\title{
Eficacia de Resecciones Hepáticas por Metástasis de Origen Colorrectal
}

\section{Efficacy of Hepatic Resections for Metastases of Colorectal Origin}

*Correspondencia:

yukito_music@hotmail.com

Teléfono [593] 0993127194

Conflicto de intereses: Los autores declaran no tener conflictos de intereses.

Fondos: Ver la página 51

Recibido: 9 Enero 2019

Aceptado: 21 Marzo 2019

Publicado: 30 Abril 2019

Membrete bibliográfico: Panchana G, Macías P, Zambrano D, Lara M, Idrovo L, Panchana G. Eficacia de Resecciones Hepáticas por Metástasis de Origen Colorectal. Rev. Oncol. Ecu 2019;29(1):45-53.

ISSN: $2661-6653$

DOI: https://doi.org/10.33821/284

Copyright Panchana, et al. Este artículo es distribuido bajo los términos de Creative Commons Attribution License, el cual permite el uso y redistribución citando la fuente y al autor original.

\section{Guido Panchana Egüez ${ }^{1-2}$, Pamela Macías Fernández ${ }^{*}$ (D), Diego A. Zambrano2-3 Luis Mauricio Lara Perlaza1 (D), Luis Idrovo Murillo1, Guido Panchana Coello4}

1. Servicio de Cirugía, SOLCA - Guayaquil.

2. Universidad de Especialidades Espíritu Santo (UEES), Guayaquil-Ecuador.

3. Association Alsace Andes - Post IRCAD Program, Estrasburgo-Francia.

4. Facultad de Medicina, Universidad Católica Santiago de Guayaquil, GuayaquilEcuador.

\section{Resumen}

Introducción: El cáncer colorrectal (CCR) es la segunda neoplasia más común después del cáncer gástrico, y con frecuencia desarrolla metástasis hepática. Se presenta un estudio unicéntrico del abordaje quirúrgico, resultados y sobrevida pacientes sometidos a cirugía más tratamiento sistémico de metástasis hepáticas de CCR.

Métodos: Se realizó un estudio retrospectivo y descriptivo en el Instituto Oncológico del Ecuador de Solca- Guayaquil desde enero del 2015 a diciembre del 2018. Se incluyeron todos los pacientes del Servicio de Laparoscopía operados por cáncer colorrectal, como diagnóstico inicial, que durante sus controles periódicos presentaron metástasis hepáticas y fueron candidatos para tratamiento quirúrgico de metastasectomias, previa valoración con comités de tumores de tubo digestivo.

Resultados: La muestra final incluyó 6 pacientes a quienes se les pudo realizar metastasectomías hepáticas anatómicas y no anatómicas. La edad media fue 60 años, con predominio del sexo femenino $(n=4,66,6 \%)$. Las lesiones hepáticas se presentaron en un solo segmento hepático en el $66 \%$ a las que se les realizo resección total, y cuyas complicaciones presentadas fueron del $33 \%$, que se resolvieron durante la estancia hospitalaria. Un paciente recibió neoadyuvancia, y solo un paciente presentó metástasis metacrónica. La sobrevida es del 100\% hasta la finalización del estudio.

Conclusión: Las decisiones multidisciplinarias sobre una oportuna indicación de metastasectomía hepática, disminuyen la morbimortalidad e incrementan la sobrevida de los pacientes con metástasis hepáticas de cáncer colorrectal. 
Palabras Claves: NEOPLASIAS COLORRECTALES, METÁSTASIS DE LA NEOPLASIA, HÍGADO, NEOPLASIAS HEPÁTICAS, HEPATECTOMÍA, CIRUGÍA GENERAL

DOI: $10.33821 / 284$

\section{Abstract}

Introduction: Colorectal cancer (CRC) is the second most common neoplasm after gastric cancer, and with the frequency of liver metastases. We present a unicentric study of the surgical approach, results and survival, patients, surgery and systemic treatment of hepatic metastases of CRC.

Methods: A retrospective and descriptive study was carried out at the Oncology Institute of Ecuador in Solca- Guayaquil from January 2015 to December 2018. All patients of the Laparoscopic Service operated by colorectal cancer were included as initial diagnosis, which during their controls newspapers presented liver metastases and were candidates for surgical treatment of metastasectomies, previous valuation with digestive tract tumors committees.

Results: The final sample included 6 patients who could undergo anatomical and non-anatomical hepatic metastasectomies. The average age was 60 years, with a predominance of females $(n=4$, $66.6 \%$ ). Hepatic lesions were present in a single hepatic segment in $66 \%$ of those who underwent total resection, and whose complications were $33 \%$, which were resolved during the hospital stay. One patient received neoadjuvant therapy, and only one patient presented metachronous metastasis. Survival is $100 \%$ until the end of the study.

Conclusion: Multidisciplinary decisions on a timely indication of hepatic metastasectomy reduce morbidity and mortality and increase the survival of patients with hepatic metastases of colorectal cancer.

Keywords: COLORECTAL NEOPLASMS, NEOPLASM METASTASIS, LIVER, LIVER NEOPLASMS, HEPATECTOMY, GENERAL SURGERY

DOI: $10.33821 / 284$

\section{Introducción}

El cáncer colorrectal es el de mayor incidencia en España alcanzando un 15 \% en el año 2012 [1]. En Ecuador, según cifras publicadas por Solca, representa una incidencia de $9.7 \times 100$ mil habitantes en mujeres y de 7.9 × 100 mil habitantes en hombres.

A nivel mundial el cáncer colorectal que supuso en 2004 el $1.1 \%$ de todas las muertes, la cual constituye la vigésima posición de causa de muerte global, lo que está previsto que aumente en el año 2030 hasta un $1.4 \%$, situándose en la posición decimocuarta [2, 3]. El hígado es el principal órgano donde se produce metástasis tras el desarrollo del cáncer colorectal, hasta un $50-60 \%$ de los pacientes la padecen a lo largo de la evolución de la enfermedad, de hecho entre un $15-25 \%$ el diagnóstico es simultáneo, mientras que en el resto aparece entre los 3-4 años siguientes [4-5].

En estos casos la resección del hepática es el tratamiento más eficaz, aumentando la supervivencia del individuo hasta los 5 años en el $50 \%$ de los casos, incluso hasta los 10 años entre el 17-25 \% [6-7], y consiguiendo disminuir la mortalidad entre $1 \%-2 \%$ [8, 9]. Aun 
así no todos los pacientes son susceptibles de esta resección, y su correcta elección es muy importante para el verdadero éxito de la intervención. En los últimos años los criterios de inclusión se han incrementado $[10,11]$, hasta que la única premisa para la resección es que sea posible extirpar toda la masa tumoral con un margen libre de seguridad y un remanente hepático suficiente con buena capacidad funcional [10]. Aunque las resecciones hepáticas se realizan con intención curativa, el $60 \%$ de los pacientes operados por MHCCR desarrollarán enfermedad recurrente en los 2 años siguientes a la misma [11]. Entre el 20$30 \%$ de los pacientes que sufren recurrencia después de la primera resección hepática tendrá una enfermedad que potencialmente permite una rehepatectomía con los mismos criterios de inclusión que para la resección inicial [12]. El objetivo del presente estudio es presentar una serie de casos de pacientes con el diagnóstico de metástasis hepáticas de origen colorrectal sometidas a hepatectomía.

\section{Materiales y Métodos}

El presente estudio observacional, retrospectivo de cohorte transversal, se realizó en el Departamento de Cirugía del Instituto Oncológico Nacional “Dr. Juan Tanca Marengo" SolcaGuayaquil. Ingresaron al estudio todos los registros de las pacientes con diagnóstico de Cáncer de Colorrectal que fueron sometidos a Hepatectomía en el período comprendido desde enero del 2015 a diciembre del 2018.

El universo estuvo conformado de todos los pacientes que ingresaron al servicio con tumores colorrectal y metástasis hepática y que fueron considerados para tratamiento quirúrgico con hepatectomía que tuvieron todos los datos analizables. El muestreo fue no probabilístico de tipo intencional.

Se consideró la información de historias clínicas del sistema de INTRANET que utiliza el Instituto Oncológico Nacional-Solca. Se realizó una búsqueda de paciente que se hayan sometido en los últimos 4 años a resecciones hepáticas anatómicas o no anatómicas por presentar metástasis de origen colorrectal. Se realizó una selección adecuada de pacientes con estas características y que cumplan los criterios de inclusión antes mencionados. Si el paciente con metástasis no tenía criterios de resecabilidad, se empezaba con la realización de neoadyuvancia para determinar su resecabilidad post tratamiento quimioterápico. 
Los criterios quirúrgicos para hepatectomía fueron los siguientes:

Criterios quirúrgicos de inclusión

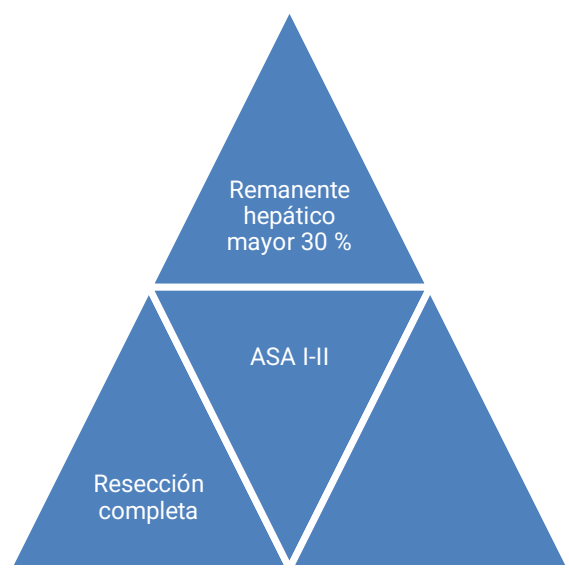

Criterios de exclusión

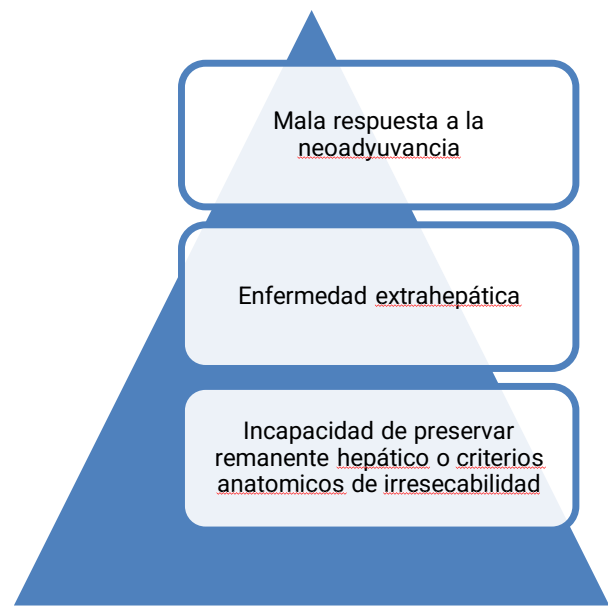

\section{Resultados}

En el periodo de estudio se evaluaron 6 pacientes con diagnóstico de metástasis secundarios a cáncer de colon rectal, a quienes se les realizo resecciones hepáticas anatómicas y no anatómicas (Figura 1-3). En la muestra se observó una edad media de 50 años en un rango entre 40 a 60 años, y relación de sexo con predominio al femenino en $66.6 \%$ (n.4) (Tabla 1).

Tabla 1. Pacientes incluidos en el estudio.

\begin{tabular}{|l|l|c|c|c|c|c|}
\hline Caso & Sexo & Edad & $\begin{array}{l}\text { Metástasis } \\
\text { unilaterales }\end{array}$ & $\begin{array}{l}\text { Metástasis } \\
\text { bilaterales }\end{array}$ & $\begin{array}{l}\text { Número de } \\
\text { metástasis }\end{array}$ & Neoadyuvancia \\
\hline $\mathrm{A}$ & Masculino & 56 & $\mathrm{Si}$ & - & 3 & - \\
\hline $\mathrm{B}$ & Masculino & 54 & $\mathrm{Si}$ & - & 1 & - \\
\hline $\mathrm{C}$ & Femenino & 58 & $\mathrm{Si}$ & - & 2 & - \\
\hline $\mathrm{D}$ & Femenino & 49 & - & $\mathrm{Si}$ & 4 & $\mathrm{Si}$ \\
\hline $\mathrm{E}$ & Femenino & 48 & $\mathrm{Si}$ & - & 1 & - \\
\hline $\mathrm{F}$ & Femenino & 50 & - & $\mathrm{Si}$ & 3 & - \\
\hline
\end{tabular}

Las complicaciones post quirúrgicas de la metastasectomías las más frecuentes, que se presentaron en el $33 \%$ de los casos, un paciente presento neumonía nosocomial la misma que se trató con antibioticoterapia con buena respuesta sin internación, el segundo paciente se presentó con infección de la herida quirúrgica a las 48 horas posteriores a la cirugía, siendo tratada con curaciones diarias y buena resolución (Tabla 2). 
Tabla 2. Complicaciones postquirúrgicas frecuentes.

\begin{tabular}{|l|c|}
\hline Tipo de complicación & Frecuencia \\
\hline Neumonía & $1 / 6$ \\
\hline Infección de Herida Quirúrgica & $1 / 6$ \\
\hline
\end{tabular}

Lo referente a la estancia hospitalaria, el promedio fue de 5 a 7 días; se pudo constatar que de estos días, los primeros 2 fueron exclusivamente de estancias en unidad de cuidados intensivos y los días posteriores fueron de estancia en sala general.

El seguimiento actualmente es de 48 meses, los pacientes son controlados en consulta cada mes durante los primeros 3 meses y posteriormente cada 3 meses durante los primeros 3 años. Con exámenes de gabinete como TAC de abdomen y pelvis con contraste oral e intravenoso anuales y controles de marcadores tumorales en cada consulta.

El seguimiento demostró que solo 1 paciente que corresponde al $15 \%$ de la muestra total, presento una metástasis meta crónica post resección. La sobrevida de los pacientes a la fecha actual es del $100 \%$ con una calidad de vida aceptable y sin signos de insuficiencia hepática.

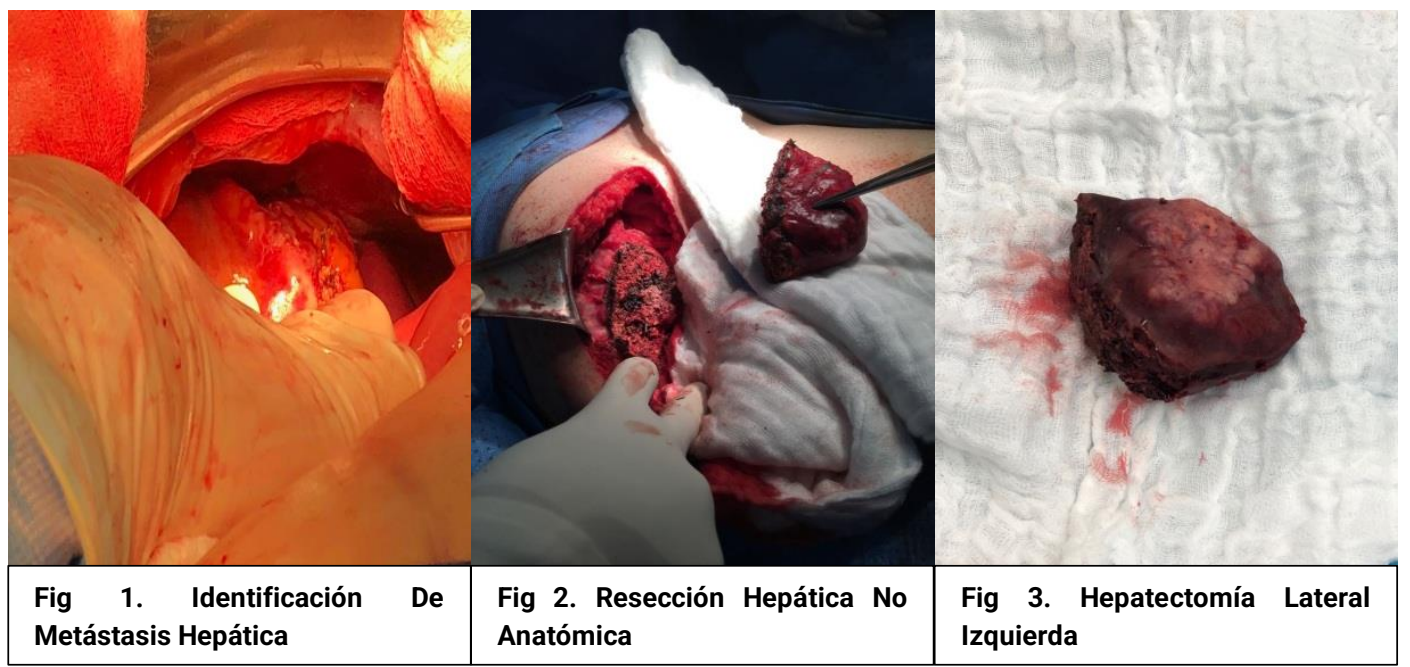

\section{Discusión}

En la presente serie, los pacientes afectos de cáncer color rectal que desarrollaron metástasis hepáticas. La cirugía de resección hepática ha demostrado ser el tratamiento ideal para la supervivencia de los pacientes intervenidos quirúrgicamente por metastasectomías. La creación de grupos multidisciplinarios y equipos de cirujanos con especial dedicación a la cirugía digestiva, tal como existe en nuestro instituto oncológico, es la mejor estrategia para una selección adecuada de los pacientes; los resultados en nuestra series tuvieron resultados similares a los mejores series extranjera, con una morbilidad de alrededor de $30 \%$ y una mortalidad de $3 \%[1,4]$. 
Sobre el manejo del tipo de resecciones hepáticas locales o no anatómicas [4], existen pocos trabajos que comparen ambas técnicas en forma adecuada. Las resecciones anatómicas tendrían los beneficios de lograr un mejor margen y que pueden incluir metástasis en la pieza quirúrgica no pesquisadas previamente, con una morbilidad similar. En nuestro estudio se realizó resección no anatómica

El sangrado quirúrgico es más controlado, debido a que se siguen planos anatómicos en la resección hepática [1,2-4]. Las resecciones no anatómicas permiten preservar una mayor cantidad de parénquima hepático sano, lo que es de especial relevancia en pacientes con fibrosis o esteatosis hepática mayor.

En esta serie realizamos resecciones locales unilaterales en $66.6 \%$ de los pacientes, el manejo quirúrgico permitió dejar libre de enfermedad a pacientes con metástasis hepáticas unilaterales y bilaterales, preservando un adecuado volumen hepático residual. Ninguno de los pacientes presento disfunción hepática post resección.

La quimioterapia en pacientes con Metástasis Hepáticas con Cáncer Colorrectal, ha probado ser efectiva como estrategia neoadyuvante [4-8]. Existen dudas sobre cuál es el mejor esquema de quimioterapia. En el estudio solo 1 paciente se le realizo quimioterapia de segunda línea.

La serie estudiada permite confirma que la resección de Metástasis Hepática por Cáncer Colorrectal es una terapia segura que permite la curación de una enfermedad neoplásica avanzada. La resección quirúrgica de las metástasis hepáticas es la única forma de tratamiento potencialmente curativa; actualmente la mayoría de los informes demuestran que la morbilidad y mortalidad operatoria es baja y los resultados a largo plazo son mucho mejores que en los pacientes no tratados. El éxito dependerá de la forma de selección de los casos que debería ser realizado por comités multidisciplinario en manejo de patología digestivas hepáticas.

\section{Conclusiones}

En esta serie con resecciones locales unilaterales en su mayoría, se preservó un volumen hepático residual en la cual ningún paciente presentó disfunción hepática post resección. Las complicaciones fueron menores al 33 \% y la supervivencia del grupo es de 48 meses.

\section{Agradecimientos}

Reconocemos a las personas que participaron indirectamente en el estudio tales como el personal técnico, pacientes y personal del Instituto Oncológico Nacional "Dr. Juan Tanca Marengo", Solca Guayaquil. 


\section{Información adicional}

Abreviaturas

MHCC: Metástasis Hepática Colorectal.

SOLCA: Sociedad de Lucha contra el Cáncer.

Nota del Editor

La Revista Oncología Ecu

permanece neutral con

respecto a los reclamos

jurisdiccionales en mapas

publicados y afiliaciones

institucionales.

\section{Archivos Adicionales}

Ninguno declarado por los autores.

Fondos

Los fondos de la investigación fueron propios de los autores del presente artículo.

\section{Disponibilidad de datos y materiales}

Existe la disponibilidad de datos bajo solicitud al autor de correspondencia. No se reportan otros materiales.

\section{Contribuciones de los autores}

GPE, PMF, DAZ realizaron la idea de investigación, revisión bibliográfica. MLP, LIM, GPC recolección de datos, escritura del artículo. GPE realizó el análisis crítico del artículo. PMF realizó las correcciones editoriales. Todos los autores leyeron y aprobaron la versión final del artículo.

Aprobación de ética y consentimiento para participar

No aplica a este estudio.

Consentimiento para publicación

No aplica.

\section{Información de los autores}

Guido Antonio Panchana Eguez, Cirujano Oncólogo. Jefe del departamento de Laparoscopía del ION SOLCA. Docente de la Facultad de Postgrado Universidad de Especialidades Espíritu Santo. Guayaquil. Ecuador. 


\section{¿@panchanag@gmail.com 0999748249}

Pamela Katherine Macias Fernandez, Cirujana general, miembro del departamento de Cirugía Oncológica y departamento de Laparoscopía del ION SOLCA.

@ yukito_music@hotmail.com 0993127194

iD https://orcid.org/0000-0002-8047-8148

Diego A. Zambrano, Médico del departamento de Cirugía, Instituto Oncológico Nacional del Ecuador "Dr. Juan Tanca Marengo" -SOLCA, Guayaquil-Ecuador. Association Alsace Andes - Post IRCAD Program, Estrasburgo-Francia. Universidad de Especialidades Espíritu Santo (UEES), Guayaquil-Ecuador.

@ dizampe@gmail.com (593)-4-371-8300 ext. 2123

Luis Mauricio Lara Perlaza, Cirujano Oncólogo, miembro del departamento de Cirugía Oncológica y departamento de Cirugía Digestiva del ION SOLCA.

@@llaraperlaza@hotmail.com 0997585589

Luis Idrovo Murillo, Departamento de Cirugía, Instituto Oncológico Nacional del Ecuador “Dr. Juan Tanca Marengo" -SOLCA, Guayaquil-Ecuador.

Guido Arturo Panchana Coello,

@guidopanchana93@gmail.com @ 0995116848

Revisiones por pares

Acceda a la revisión de pares académicos en el siguiente enlace: https://publons.com/review/4526853/

\section{Referencias}

Abreviaturas en la referencias DOI: Digital Object Identifier PMID: PubMed Identifier SU: Short URL
1. Dávila D, Palacios 0, Naranjo C. Metástasis hepáticas en el Cáncer Colorrectal: Estrategia terapéuticas y recomendaciones actuales. Rev Colombiana Cir. 2017;32:304-18. SU: bit.ly/2R9ISrU

2. Siegel RL, Miller KD, Jemal A. Cancer Statistics, 2017. CA Cancer J Clin. 2017;67:7-30. DoI: $10.3322 /$ caac. 21387

3. Uribe M, Paqui L, Uribe-Echevarría S, Romanque P, Azabache V. Resección de metástasis hepáticas en cáncer de colon. Nuevas perspectivas. Gastroenterol Latinoam. 2014;25:50-3. SU: bit.ly/2IxfgAA

4. Díaz J, Castillo J, Csendes A, Sauré A. Resección de metástasis Hepáticas en Cáncer Gástrico: Experiencia Preliminar. Rev Chi Clr 2013;65(6):520-524. DOI: 10.4067/S0718-40262013000600008

5. Navarro F, Navarro P, Miron B, Delgado M, Jimenez J, Garcia P, et al. Recurrencia de la Cirugía de las Metástasis Hepáticas del Cáncer Colorrectal y repetición de la Resección. Rev Esp Enferm Dig 2015;107(12):732-739. SU: bit.ly/2lzoZqg 
6. Norero E, Jarufe N, Butte JM, Norero B, Duarte I, Torres J, et al. Resultados del tratamiento quirúrgico de las metástasis hepáticas por cáncer colorrectal. Rev. méd. Chile 2009;137(4):487-496. SU: bit.ly/2KMxPUq

7. Perez B, Palomeque A, Navarro P, Gonzalez A, Navarro F. Metástasis Hepáticas de Orígen Colorrectal Sincrónicas: ¿ Intervención Simultanea o Secuencial?. Revista Chilena de Cirugia, Cir. 2015;67(2): 158166. SU: bit.ly/2IBYbWp

8. Penna C. Prise en charge des patients ayant un cancer du foie. Les metastases hépatiques des cancers colo-rectaux. Bull Cancer 2003;90:79-83. SU: bit.ly/2F6uCvh

9. Beguiristain A, Gisasola E, Ruiz I, Jiménez R, Arteaga X, Arriaga M. Protocolo de actuación para el manejo del paciente con metástasis hepáticas de adenocarcinoma de colon-recto 49 , Comité Tumores hepatobiliopancreáticos, Hospital Universitario Donostia - 2012; 1-15. SU: $\underline{\text { bit.ly/31tzp3n }}$

10. Akgül O, Çetinkaya E, Ersöz S, Tez M. Role of surgery in colorectal cancer liver metastases. World J Gastroenterol. 2014;20(20):6113-22. DOI: 10.3748/wjg.v20.i20.6113

11. Tsalis k, Loannidis 0 , Cheva A, Antigoni N, Antoniou N, Parpoudi S, et al. A 20-year single center experience in the surgical treatment of colorectal liver metastasis, JBUON 2018; 23(6):1640-1647. SU: bit.ly/2WArgXd

12. Chiappa A, Bertani E, Zbar A, Foschi D, Fazio N, Zampino M, et al. Optimizing treatment metastases from colorectal cancer: Resection or resection plus ablation? Int J Oncol 2016;48(3):1280-9. DOI: 10.3892/ijo.2016.3324. 